\title{
Efecto de las variables experimentales sobre la microdureza en aleaciones Al-6Si-3Cu-xMg T6
}

\author{
ALFONSO, I. ' ; MALDONADO, C. ${ }^{\text {II }}$; GONZÁLEZ, J.G. ${ }^{\text {I }}$ MEDINA, A. ${ }^{\text {II }}$ \\ ${ }^{\mathrm{I}}$ Instituto de Investigaciones en Materiales, Universidad Nacional Autónoma de México. \\ Circuito Exterior, Cd. Universitaria, Del. Coyoacán, México, DF. México. C.P. 04510 \\ e-mail: ialfonso@iim.unam.mx, joseggr@servidor.unam.mx

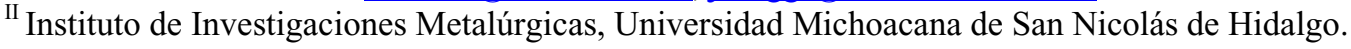 \\ Morelia, Michoacán. México. C.P. 58000. \\ e-mail: cmzepeda@zeus.umich.mx, ariosto@jupiter.umich.mx
}

\begin{abstract}
RESUMEN
Tres aleaciones cuaternarias $\mathrm{Al}-6 \mathrm{Si}-3 \mathrm{Cu}-\mathrm{xMg}(\mathrm{x}=0.59,3.80$ y $6.78 \%$ en peso) fueron producidas utilizando fundición convencional de lingotes y solidificación rápida mediante "melt-spinning". El estudio se concentró en investigar el efecto del contenido de $\mathrm{Mg}$ y del tiempo y la temperatura de envejecido sobre los precipitados y la microdureza. Las aleaciones se sometieron a tratamientos térmicos $\mathrm{T} 6$, consistentes en calentar las muestras a $480{ }^{\circ} \mathrm{C}$ por $12 \mathrm{~h}$ y envejecido a 150,180 y $210{ }^{\circ} \mathrm{C}$, durante tiempos comprendidos entre 0.05 y 100 horas. Se aplicaron diseños multifactoriales, resumidos en la dependencia $\mathrm{VHN}=\mathrm{f}(\mathrm{t}, \mathrm{T}$, $\% \mathrm{Mg}$ ), donde VHN es la microdureza Vickers, t es el tiempo y $\mathrm{T}$ la temperatura de envejecido. Se utilizaron técnicas de microscopía electrónica de transmisión y microdureza. Los precipitados obtenidos fueron diferentes: $\mathrm{CuAl}_{2}$ en forma de aguja para las aleaciones con $0.59 \%$ de $\mathrm{Mg}$; y Q $\left(\mathrm{Al}_{5} \mathrm{Cu}_{2} \mathrm{Mg}_{8} \mathrm{Si}_{6}\right)$ irregulares para las aleaciones con 3.80 y $6.78 \%$ de $\mathrm{Mg}$. Para las aleaciones obtenidas por solidificación rápida, el factor que más influyó sobre la microdureza fue la temperatura, seguido por el contenido de $\mathrm{Mg}$ y el tiempo de envejecido. El aumento del contenido de $\mathrm{Mg}$ origina mayores microdurezas. Para las aleaciones obtenidas por solidificación convencional la temperatura es el único factor que provoca cambios significativos sobre la microdureza.
\end{abstract}

Palabras clave: Melt-spinning, aleaciones de aluminio, magnesio, envejecido, precipitación, recristalización.

\section{Effect of the experimental variables on the Al-6Si-3Cu-xMg T6 microhardness alloy}

\section{ABSTRACT}

Three Al-6Si-3Cu-xMg (x = 0.59, 3.80 and 6.78 wt. \%) alloys were produced using conventional ingot casting metallurgy and melt-spinning. The study was focused to investigate the effect of Magnesium content and aging time ant temperature on precipitates and microhardness. Obtained alloys were solution heat treated at $480^{\circ} \mathrm{C}$ for $12 \mathrm{~h}$ and aged at 150,180 and $210^{\circ} \mathrm{C}$ for times between 0.05 and $100 \mathrm{~h}$. Multifactorial designs were used, summarized in the dependency: $\mathrm{VHN}=\mathrm{f}(\mathrm{t}, \mathrm{T}, \% \mathrm{Mg})$, where VHN is Vickers microhardness, $\mathrm{t}$ is aging time and $\mathrm{T}$ is aging temperature. Transmission Electron Microscopy and microhardness techniques were used. Results shown variations in precipitates composition: $\mathrm{CuAl}_{2}$ needles for the alloys with $0.59 \% \mathrm{Mg}$ and $\mathrm{Q}\left(\mathrm{Al}_{5} \mathrm{Cu}_{2} \mathrm{Mg}_{8} \mathrm{Si}_{6}\right)$ with irregular shapes, for the alloys with 3.80 and $6.78 \%$ $\mathrm{Mg}$. For melt-spinning alloys, temperature originates the most significant variation on microhardness, followed by $\mathrm{Mg}$ content and aging time. The increase of $\mathrm{Mg}$ content originates greater microhardnesses. For conventionally cast alloys temperature is the only factor that significantly changes microhardness.

Keywords: Melt-spinning, aluminum alloys, magnesium, aging, precipitation, recrystallization. 


\section{INTRODUCCIÓN}

Las aleaciones Al-Si-Cu-Mg son muy utilizadas en la industria automotriz por su alta relación resistencia-peso. Estas aleaciones tienen una resistencia media entre las Al-Si y las Al-Cu, lo cual hace que sean útiles en aplicaciones donde la resistencia requerida no sea muy alta. El Si imparte elevada fluidez y el $\mathrm{Cu}$ aumenta la resistencia mecánica [1]. El mecanismo por el cual la resistencia se incrementa es la formación de precipitados de tipo coherente con la matriz de Al. Para la aleación ternaria Al-Si-Cu el precipitado $\mathrm{Al}_{2} \mathrm{Cu}$ es el encargado de aumentar la dureza, pero al adicionar $\mathrm{Mg}$ precipita el $\mathrm{Mg}_{2} \mathrm{Si}$, el cual, junto con el $\mathrm{Al}_{2} \mathrm{Cu}$, origina mayores aumentos en la dureza y la resistencia $[\underline{1}, \underline{2}, \underline{3}]$. Entre estas aleaciones cuaternarias está la 319, que presenta un contenido de $\mathrm{Si}$ entre 5.5 y $6.5 \%$ en peso, $\mathrm{Cu}$ en el rango de 3.0 a $4.0 \%$ y $\mathrm{Mg}$ para fines de reforzamiento, pero a expensas de la disminución de la ductilidad, no sobrepasando el $1 \%[\underline{3}, \underline{4}, \underline{5}]$. No obstante se encontró reportada una patente acerca de una aleación de aluminio similar a la 319 , pero con contenidos de magnesio entre 0.5 y $7.0 \%$ en peso [] ]. Este tipo de aleaciones presenta como una de sus principales cualidades una alta resistencia. El $\mathrm{Mg}$ aumenta la dureza y resistencia por solubilización sólida.

Para obtener la máxima dureza a estas aleaciones se les aplica un tratamiento térmico que consiste en un tratamiento de solubilización de las segundas fases, seguido de un envejecimiento, proceso necesario para la precipitación controlada de los constituyentes endurecedores. Este tratamiento es particularmente eficaz para aleaciones con alto contenido de $\mathrm{Mg}$, por la precipitación del $\mathrm{Mg}_{2} \mathrm{Si}$. Ouellet et al. [7] reportan que el pico de endurecimiento aparece por precipitación cooperativa entre partículas de $\mathrm{Al}_{2} \mathrm{Cu} \mathrm{y} \mathrm{Mg}_{2} \mathrm{Si}$. No obstante se ha encontrado que para aleaciones cuaternarias el proceso de precipitación es más complejo, precipitando fases diferentes a las antes mencionadas []]. La estructura fundida para las aleaciones 319 incluyen la matriz $\alpha$-aluminio, el eutéctico $\mathrm{Al}-\mathrm{Si}$, partículas de $\mathrm{Si}, \mathrm{Mg}_{2} \mathrm{Si}, \mathrm{Al}_{2} \mathrm{Cu}, \mathrm{Al}_{5} \mathrm{Cu}_{2} \mathrm{Mg}_{8} \mathrm{Si}_{6}$ y otros intermetálicos complejos $[\underline{7}, \underline{8}, \underline{9}, \underline{10}]$.

Las relaciones entre los elementos de aleación son muy importantes en los procesos de precipitación. Cuando la relación $\mathrm{Cu}: \mathrm{Mg}$ es cercana a 2.2 precipita principalmente el $\mathrm{Mg}_{2} \mathrm{Si}(\beta)$, mientras que al acercarse a 8 es predominante $\theta^{\prime}$, un predecesor de la fase $\mathrm{CuAl}_{2}$ [4]. Utilizando solidificación rápida es posible aumentar la cantidad de elementos en solución, modificando las relaciones entre elementos de aleación [11], y por ende los precipitados formados. Además por este método se pueden obtener algunos componentes del automóvil como son barras de conexión, mangas de cilindros, retenedores de pistones y válvulas y partes del compresor. Cuando las velocidades de enfriamiento son mayores a $10^{3} \mathrm{~K} / \mathrm{s}$, las aleaciones obtenidas presentan características como la reducción en tamaño de grano, extensión de la solubilidad sólida, menor segregación y en algunos casos las formación de fases metaestables cristalinas y amorfas [11, 12]. El uso de "melt-spinning" para producir aleaciones de aluminio a velocidades de enfriamiento que exceden $10^{6} \mathrm{~K} / \mathrm{s}$, resulta en la obtención de cintas con propiedades mecánicas superiores a las obtenidas por el procedimiento convencional.

Existen algunos trabajos acerca de la caracterización de aleaciones Al-Si-Cu-Mg con bajos contenidos de $\operatorname{Mg}[\underline{6}, \underline{7}, \underline{8}, \underline{9}, \underline{10}]$ obtenidas por métodos convencionales, pero la información para aleaciones con alto contenido de $\mathrm{Mg}$ es escasa. De igual manera existe poca información acerca de la caracterización de aleaciones $\mathrm{Al}-\mathrm{Si}-\mathrm{Cu}-\mathrm{Mg}$ obtenidas por solidificación rápida y el proceso de precipitación que experimentan [11]. Por tal motivo el presente estudio tiene como objetivo principal el análisis del efecto del $\mathrm{Mg}$ y del modo de solidificación sobre la respuesta a los tratamientos térmicos de envejecido en aleaciones cuaternarias Al$\mathrm{Si}-\mathrm{Cu}-\mathrm{Mg}$. Para este propósito se utilizaron técnicas de Microscopía Electrónica de Transmisión y Microdureza. Mediante el uso de estas técnicas, en el presente trabajo será posible dilucidar aspectos que no se han encontrado reportados en la literatura, como es el caso de los precipitados que se forman al aumentar el contenido de Mg. Para disminuir el número de experimentos y tener una mejor idea de la influencia de las variables experimentales sobre las propiedades de las aleaciones obtenidas, se empleó diseño experimental multifactorial, obteniéndose las dependencias $\mathrm{VHN}=\mathrm{f}(\mathrm{t}, \mathrm{T}, \% \mathrm{Mg})$, donde VHN es la microdureza Vickers, $\mathrm{t}$ es el tiempo y T la temperatura de envejecido. Como apoyo en los cálculos se utilizó el software Statgraphics Plus 4.1.

\section{MATERIALES Y MÉTODOS}

Para la fundición de las aleaciones se utilizó como aleación base un lingote de aluminio A356 (Al8.5Si-0.3Mg). Una vez fundida, a la aleación base se le añadió $\mathrm{Cu}$ (polvo, $99.9 \%$ de pureza) y $\mathrm{Mg}$ (lingote, 99.9\%), para obtener tres aleaciones con diferentes contenidos de $\mathrm{Mg}$. La fundición se hizo en un horno de inducción Leybold-Heraeus con atmósfera controlada de $\mathrm{Ar}$, en un crisol de grafito. La temperatura de fundición se mantuvo a $750{ }^{\circ} \mathrm{C} \pm 10^{\circ} \mathrm{C}$ y se vació en moldes convencionales sin precalentamiento, dentro del horno para evitar la oxidación y la formación de poros. Las composiciones químicas de las aleaciones experimentales se muestran en la Tabla 1. Los contenidos de $\mathrm{Mg}$ añadidos fueron seleccionados tomando como base la necesidad de obtener relaciones $\mathrm{Cu}: \mathrm{Mg}$ y $\mathrm{Mg}$ :Si representativas. 
Tabla 1: Composiciones químicas en \% en peso de las aleaciones obtenidas.

\begin{tabular}{|c|c|c|c|c|c|c|c|c|}
\hline Código de la aleación & Si & $\mathbf{C u}$ & $\mathbf{M g}$ & $\mathbf{F e}$ & $\mathbf{M n}$ & $\mathbf{Z n}$ & $\mathbf{T i}$ & $\mathbf{A l}$ \\
\hline AM01 & 6.40 & 3.02 & 0.59 & 0.34 & 0.09 & 0.04 & 0.14 & Balance \\
\hline AM03 & 6.31 & 3.03 & 3.80 & 0.32 & 0.08 & 0.03 & 0.13 & Balance \\
\hline AM06 & 5.84 & 2.95 & 6.78 & 0.31 & 0.07 & 0.03 & 0.12 & Balance \\
\hline
\end{tabular}

Una parte de los lingotes obtenidos por solidificación convencional fue refundida en un crisol de cuarzo usando un horno de inducción de alta frecuencia Linn Elektronik, acoplado a un mecanismo con el cual se pueden obtener cintas por solidificación rápida mediante "melt-spinning", y que consiste en una cámara de atmósfera controlada dentro de la cual se encuentra una rueda de cobre pulida, de $200 \mathrm{~mm}$ de diámetro. Se produjeron cintas solidificadas a altas velocidades aplicando un chorro de gas (He) sobre el metal fundido, el cual sale a presión por un orificio de $0.5 \mathrm{~mm}$ de diámetro en el fondo del crisol y se solidifica al contactar la superficie de la rueda que gira a $3000 \mathrm{rpm}$. La distancia entre el crisol y la rueda fue de $7 \mathrm{~mm}$ y la velocidad tangencial $30 \mathrm{~m} / \mathrm{s}$. Las dimensiones de las cintas producidas fueron de 1-3 mm de ancho, 3-5 m de longitud y 20-60 $\mu \mathrm{m}$ de espesor.

El tratamiento térmico de solución solo se aplicó a las aleaciones obtenidas mediante fundición convencional, pues las cintas obtenidas por solidificación rápida presentan una solución sólida con mayor grado de sobresaturación debido a su modo de solidificación. La solubilización consistió en someter a las probetas a una temperatura de $480 \pm 3{ }^{\circ} \mathrm{C}$ en un horno Nabetherm de aire forzado con control automático de la temperatura, durante 12 horas. Para todos los tratamientos térmicos realizados se llevó a cabo un calentamiento lento a razón de $5{ }^{\circ} \mathrm{C}$ por minuto, esto para que la transformación de fases, y por consiguiente la formación de la solución sólida, fuese lo más cercana al equilibrio posible [1]. La temperatura de las probetas fue monitoreada usando termopares Cromel-Alumel. El temple fue realizado en agua a $60{ }^{\circ} \mathrm{C}$, temperatura recomendable debido a que disminuye la deformación del material por su menor velocidad de transferencia de calor. Se siguieron las indicaciones de la Norma B597 M1ASTM. De acuerdo a las tendencias observadas en la bibliografía se seleccionaron 3 temperaturas diferentes, que fueron 150,180 y $210^{\circ} \mathrm{C}$, mientras que los tiempos se variaron entre 0.05 y 100 horas para analizar la secuencia de precipitación.

Para la determinación de la relación entre las variables experimentales en el presente trabajo se utilizaron diseños multifactoriales, resumidos en la dependencia $\mathrm{VHN}=\mathrm{f}(\mathrm{t}, \mathrm{T}, \% \mathrm{Mg})$, donde $\mathrm{VHN}$ es la microdureza Vickers, $\mathrm{t}$ es el tiempo y $\mathrm{T}$ la temperatura de envejecido. El total de experimentos es $\mathrm{N}=\mathrm{n}^{\mathrm{k}}=3^{3}$ $=27$, donde $\mathrm{n}$ indica el número de variaciones de las condiciones de entrada $(\mathrm{n}=3) \mathrm{y} \mathrm{k}$, es el número de variables de entrada al sistema $(\mathrm{k}=3: \mathrm{t}, \mathrm{T}, \% \mathrm{Mg})$. Como apoyo para el diseño experimental se utilizó el software Statgraphics Plus 4.1.

Debido a que el tamaño de los precipitados es del orden nanométrico, es necesario el uso de Microscopía Electrónica de Transmisión (MET). Por tal motivo para el análisis de las muestras envejecidas se utilizó un microscopio FEG-Philips Tecnai F20, con filamento de emisión de campo, operado a $200 \mathrm{kV}$. Se utilizaron las técnicas de campo claro (BF) y alta resolución (HRTEM), así como difracción de electrones. Se prepararon muestras de $3 \mathrm{~mm}$ de diámetro, previamente devastadas en un Gatan Dimple Grinder, modelo 656. El objetivo de este proceso es obtener zonas delgadas para posteriormente someter a la muestra a un ataque de iones de Ar mediante PICS (Precision Ion Polishing System), utilizando un PICS Gatan modelo 691. Además se midió la microdureza, pues los cambios que se originan durante el proceso de precipitación se traducen en cambios significativos en esta propiedad. Las mediciones de microdureza se realizaron utilizando un microdurómetro Vickers Leitz Wetzlar 7556, empleando una carga de 25 g. Esta prueba está estandarizada en la norma ASTM E384-84. Se realizaron 10 mediciones por muestra para así obtener la microdureza promedio en cada condición experimental.

\section{$3 \quad$ RESULTADOS Y DISCUSIÓN}

La adición de $\mathrm{Mg}$ a la aleación 319 no causó ningún tipo de defectos de fundición, ni para las aleaciones obtenidas por fundición convencional, ni para las obtenidas por solidificación rápida. Los tratamientos térmicos tampoco originaron defectos, ni fundición de fases con bajo punto de fusión. No obstante las variables de entrada utilizadas (tiempo, temperatura y contenido de $\mathrm{Mg}$ ) para los tratamientos térmicos de envejecido sí provocaron cambios significativos en la microdureza, como puede observarse en la Figura 1a-f, correspondiente a curvas de superficie obtenidas utilizando el software Statgraphics Plus 4.1, y que permiten analizar detalladamente las combinaciones de variables para las cuales se obtienen valores máximos de microdureza. Como puede observarse los valores de microdureza son mayores para las 
aleaciones obtenidas mediante solidificación rápida, alcanzando valores entre 150 y $240 \mathrm{VHN}$, mientras que para las aleaciones obtenidas por fundición convencional solo alcanzan valores entre 100 y $160 \mathrm{VHN}$. Las causas de estas diferencias se analizarán más adelante mediante el uso de MET, y son atribuidas a la presencia de nanoestructuras en las aleaciones obtenidas por solidificación rápida. Analizando la Figura 1a-c se puede observar que para las aleaciones obtenidas por solidificación rápida y bajo contenidos de $\mathrm{Mg}(0.59$ $\%$ ), las máximas microdurezas se alcanzan para temperaturas bajas y tiempos largos, lo cual pudiera explicarse por el bajo contenido de $\mathrm{Mg}$, originando que los precipitados necesiten un mayor tiempo para alcanzar su tamaño de reforzamiento óptimo. Para contenidos de $\mathrm{Mg}$ de 3.80 y $6.78 \%$ los máximos se alcanzan para tiempos cortos, sin ser la temperatura un factor significativo. En estos casos la mayor cantidad de $\mathrm{Mg}$ provoca que desde tiempos cortos los precipitados obtengan sus características reforzantes óptimas. Para el caso de las aleaciones obtenidas por fundición convencional (ver Figura 1 d-f), para los tres contenidos de $\mathrm{Mg}$ estudiados las máximas microdurezas se obtienen para temperaturas bajas y tiempos largos, indicando que la cinética de precipitación es lenta e independiente de la temperatura, lo cual pudiera explicarse debido a las bajas cantidades de elementos de aleación en comparación con las aleaciones obtenidas por solidificación rápida.
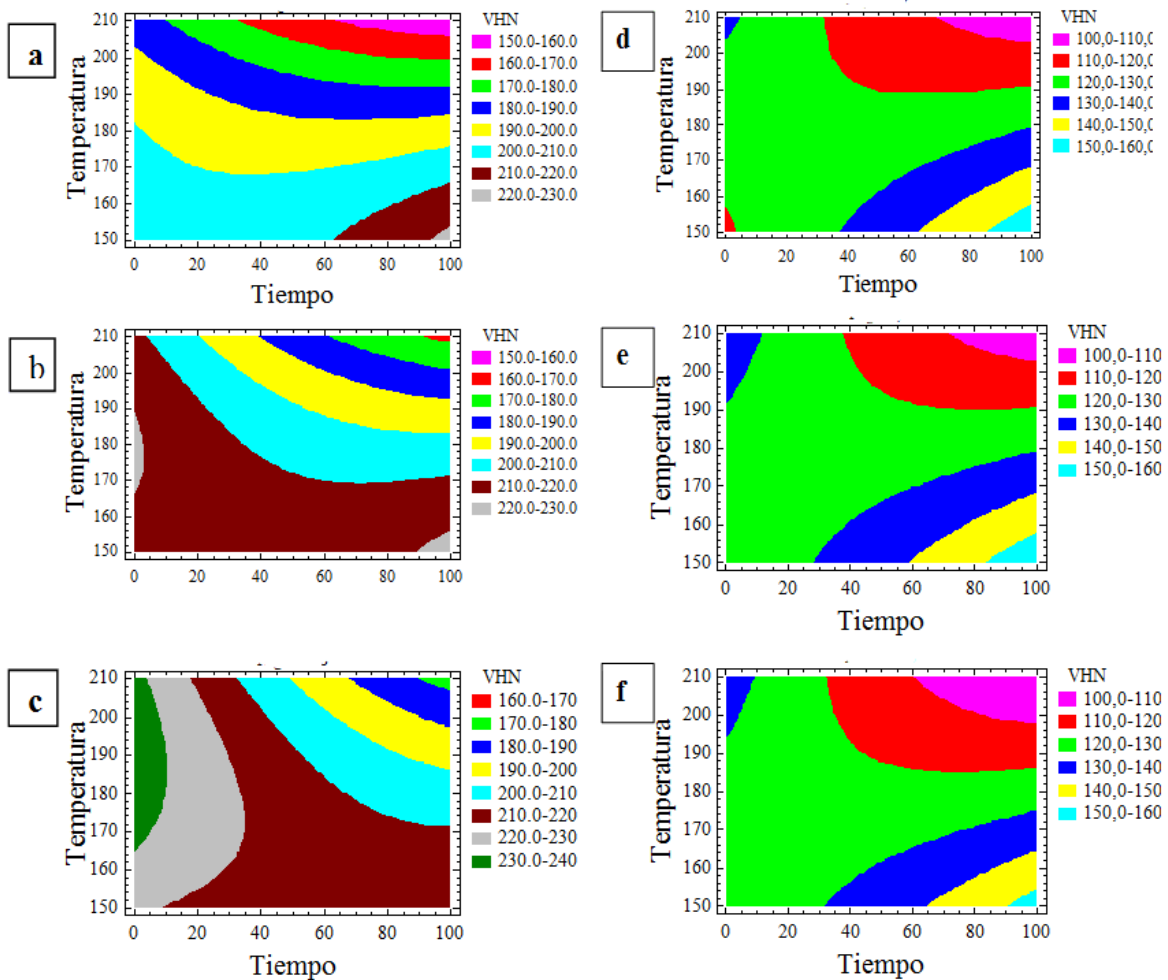

Figura 1: Influencia del tiempo y la temperatura de envejecido sobre la microdureza para las cintas obtenidas por solidificación rápida y contenidos de $\mathrm{Mg}$ de: a) $0.59 \%$, b) $3.80 \%$ y c) $6.78 \%$; y para los lingotes obtenidos mediante fundición convencional y contenidos de $\mathrm{Mg}$ de: d) $0.59 \%$ e) $3.80 \%$ y f) $6.78 \%$.

La Figura 2a-f muestra la influencia del contenido de $\mathrm{Mg}$ y del tiempo de envejecido sobre la microdureza, para diferentes temperaturas de envejecido. En el caso de las aleaciones obtenidas por solidificación rápida (Figura 2a-c) puede observarse que para una temperatura de $150{ }^{\circ} \mathrm{C}$ las máximas microdurezas se obtienen para tiempos muy largos y contenidos de $\mathrm{Mg}$ bajos, o para tiempos muy cortos y contenidos de $\mathrm{Mg}$ altos. Este comportamiento cambia al aumentar la temperatura a 180 y $210{ }^{\circ} \mathrm{C}$, pues las máximas microdurezas se obtienen para tiempos cortos y contenidos de $\mathrm{Mg}$ altos, mostrando que al incrementarse la cantidad de este elemento en tiempos cortos los precipitados formados tienen una cinética de precipitación más rápida y alcanzan su máximo reforzamiento. Para el caso de las aleaciones obtenidas por fundición convencional el comportamiento es variable. Para envejecido a $150{ }^{\circ} \mathrm{C}$ los máximos se obtienen para tiempos largos, sin influir el contenido de $\mathrm{Mg}$, mientras que para una temperatura de envejecido de $210^{\circ} \mathrm{C}$ este comportamiento se invierte, obteniéndose los máximos para tiempos cortos, lo cual está originado por el hecho de que temperaturas de envejecido altas originan que los precipitados aceleren su cinética de formación. 

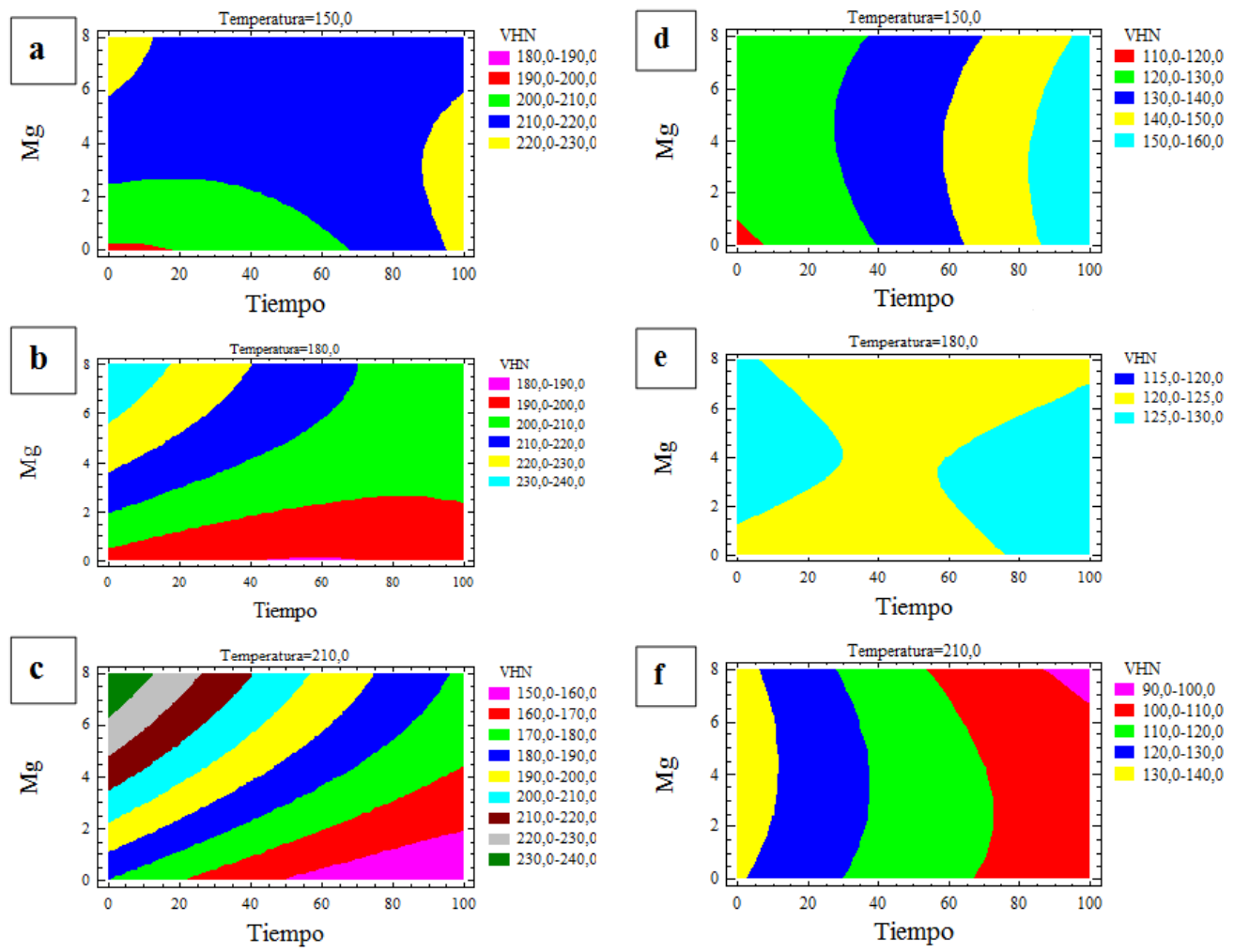

Figura 2: Influencia del contenido de $\mathrm{Mg}$ y del tiempo de envejecido sobre la microdureza para las aleaciones obtenidas por solidificación rápida envejecidas a: (a) $150^{\circ} \mathrm{C}$, (b) $180^{\circ} \mathrm{C} \mathrm{y} \mathrm{(c)} 210^{\circ}$; y por fundición convencional envejecidas a: (d) $150^{\circ} \mathrm{C}$, (e) $180^{\circ} \mathrm{C}$ y (f) $210^{\circ} \mathrm{C}$.

$\mathrm{Al}$ analizar la influencia de las variables experimentales sobre la microdureza, puede observarse que para el caso de las aleaciones obtenidas por fundición convencional (ver Figura 3a), solo la temperatura origina cambios significativos, mientras que para las aleaciones obtenidas por solidificación rápida (ver Figura $3 \mathrm{~b}$ ) las tres variables (tiempo, temperatura y contenido de $\mathrm{Mg}$ ) originan cambios significativos sobre la microdureza. Este comportamiento podría explicarse por el hecho de que el contenido de elementos de aleación en las aleaciones obtenidas por solidificación rápida es mayor, lo cual se muestra en la Tabla 2. Este mayor contenido de elementos de aleación provoca que pequeños cambios en las condiciones experimentales provoquen cambios significativos en la microdureza, debido a que los precipitados son modificados de manera importante.
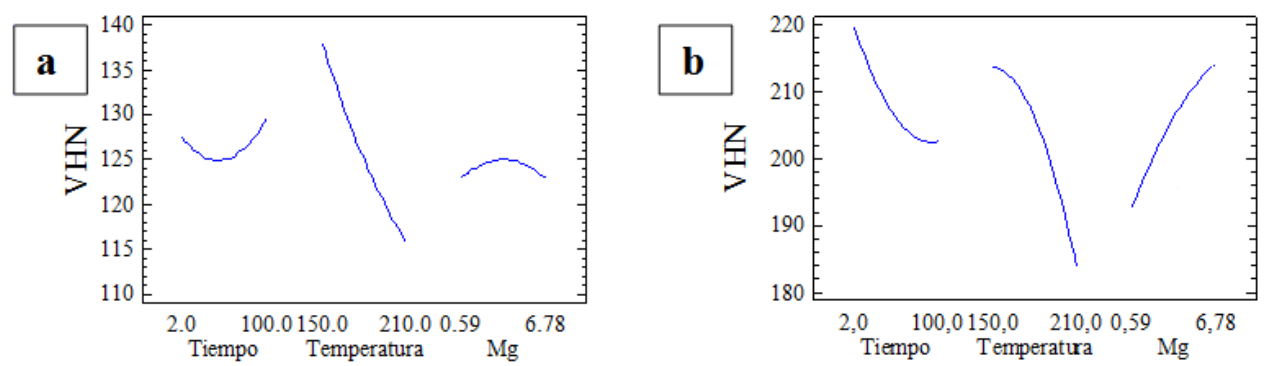

Figura 3: Efecto de las variables experimentales sobre la microdureza para las aleaciones obtenidas por fundición convencional (a) y para las aleaciones obtenidas por solidificación rápida (b). 
Tabla 2: Composición química de las soluciones sólidas y relaciones $\mathrm{Cu}: \mathrm{Mg}$ y $\mathrm{Mg}: \mathrm{Si}$ para las aleaciones experimentales.

\begin{tabular}{|c|c|c|c|c|c|}
\hline Aleación & Si & Cu & Mg & Relación Cu:Mg & Relación Mg:Si \\
\hline CAM01 & 8.97 & 1.33 & 0.25 & 5.32 & 0.03 \\
\hline CAM03 & 6.60 & 1.01 & 2.55 & 0.40 & 0.39 \\
\hline CAM06 & 4.41 & 0.95 & 6.70 & 0.14 & 1.52 \\
\hline AM01 & 1.43 & 0.26 & 0.08 & 3.25 & 0.06 \\
\hline AM03 & 0.71 & 0.73 & 0.10 & 7.30 & 0.14 \\
\hline AM06 & 0.29 & 1.46 & 0.08 & 18.25 & 0.28 \\
\hline
\end{tabular}

Para lograr comprender el comportamiento de la microdureza en estas aleaciones, se obtuvieron micrografías representativas de los precipitados. En la Figura 4a-f se pueden observar las diferencias entre los precipitados formados para las aleaciones obtenidas por los dos diferentes métodos de fundición. Las composiciones químicas de los precipitados no fueron dependientes del método de fundición, sino del contenido de $\mathrm{Mg}$, pues para las aleaciones con $0.59 \%$ de $\mathrm{Mg}$ la composición correspondió a $\mathrm{CuAl}_{2}$, mientras que para las aleaciones con medio y alto contenidos de $\mathrm{Mg}$ la composición de los precipitados fue cercana a $\mathrm{Al}_{5} \mathrm{Cu}_{2} \mathrm{Mg}_{8} \mathrm{Si}_{6}$. La única aleación que presentó precipitados de forma definida fue la que contiene $0.59 \%$ de $\mathrm{Mg}$, obtenida por fundición convencional, lo cual se corresponde con lo observado por otros investigadores para aleaciones similares, mientras que en el resto de las aleaciones se pudieron observar precipitados de formas irregulares. Estos precitados irregulares se deben a que en las soluciones sólidas sobresaturadas son mayores los contenidos de elementos de aleación, por lo que se modifica el proceso de precipitación, no presentándose la precipitación a partir de las Zonas de Guinier-Preston, que son las precursoras de los precipitados en forma de aguja. Para las aleaciones donde se obtienen los precipitados irregulares, para estadíos tempranos de la precipitación solo se observaron aglomeraciones de átomos. En el caso de las aleaciones obtenidos por fundición convencional, es de destacar que los precipitados aumentaron de tamaño a medida que aumentó el tiempo de envejecido, mientras que para el caso de las aleaciones obtenidas por solidificación rápida el comportamiento fue irregular, lo cual se explica debido a procesos de recristalización. Comparando las fracciones volumétricas (f) de los precipitados, la cantidad de éstos en las aleaciones obtenidas por solidificación rápida es mucho mayor, lo cual se debe al mayor contenido de elementos de aleación en solución sólida.
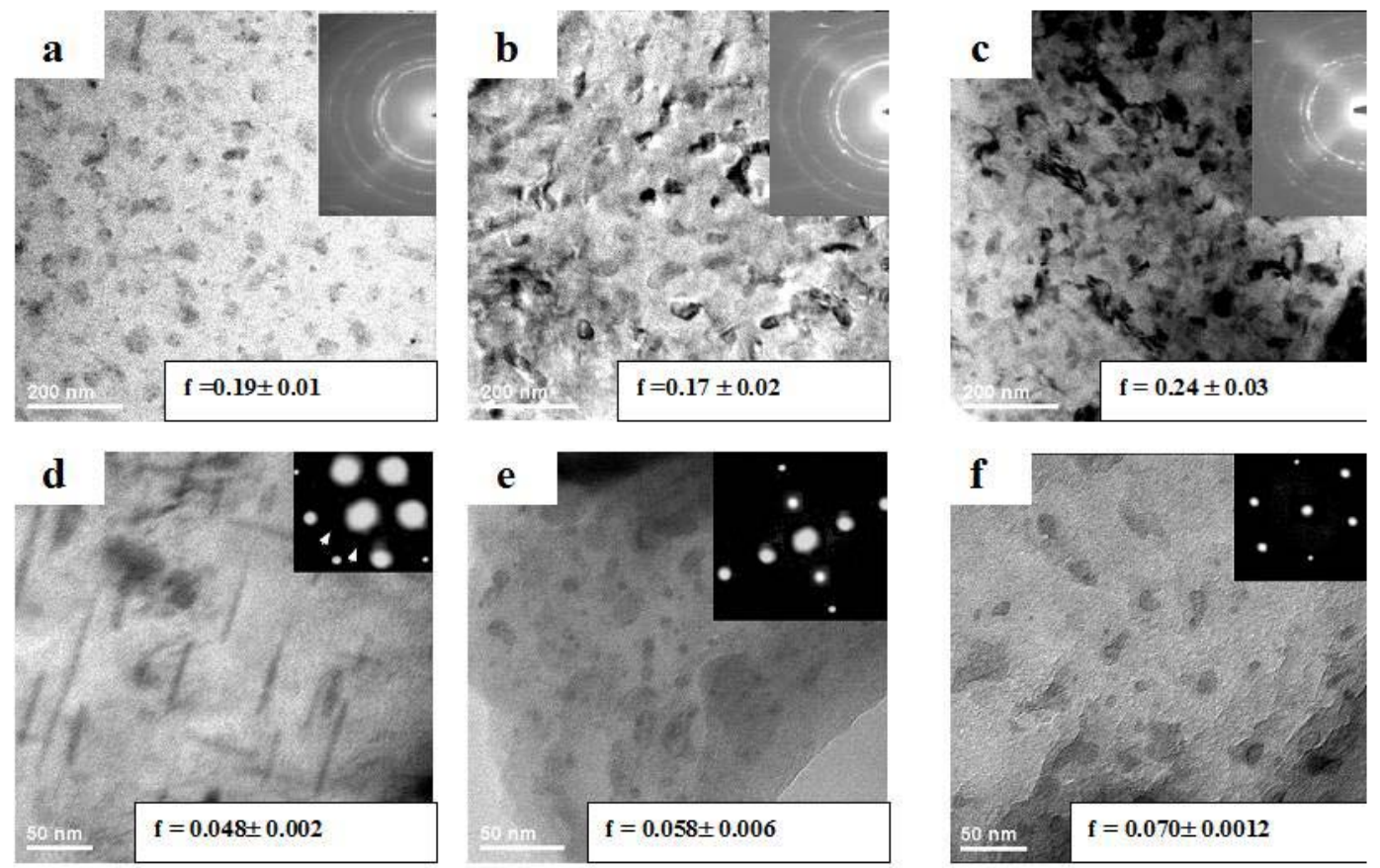

Figura 4: Micrografías obtenidas mediante MET-campo claro, y sus patrones de difracción, en las cuales se observa la forma y distribución de los precipitados para las condiciones de máxima microdureza, para las aleaciones obtenidas mediante solidificación rápida, con contenidos de $\mathrm{Mg}$ de: (a) $0.59 \%$, (b) $3.80 \% \mathrm{y}$ (c) $6.78 \%$ y para las aleaciones obtenidas mediante solidificación convencional, con contenidos de $\mathrm{Mg}$ de: (a) $0.59 \%$, (b) $3.80 \%$ y (c) $6.78 \%$. Los valores de f corresponden a las fracciones volumétricas de precipitados. 
La alta fracción volumétrica de precipitados es una de las causas de la mayor microdureza en las aleaciones obtenidas por solidificación rápida. Otra causa de la alta microdureza de las aleaciones obtenidas por solidificación rápida es la presencia de nanoestructuras, las cuales se presentaron incluso en el estado "asmelt-spun". Los patrones de difracción de las Figuras 4a-c, muestran anillos correspondientes a la fase $\alpha$-Al, denotando la existencia de cristales muy pequeños, con orientaciones diferentes, lo cual se debe al método de solidificación empleado. Nanopartículas que forman parte de estas nanoestructuras se pueden observar en la Figura 5a-f, pertenecientes a la fase $\mathrm{CuAl}_{2}$. Debido al alto contenido de elementos de aleación en estas aleaciones ocurren procesos de recristalización, que originan la formación y disolución de nanopartículas que participan de manera activa en el reforzamiento de la aleación $[\underline{12}, \underline{13}, \underline{14}]$. Para aleaciones obtenidas por solidificación rápida puede ocurrir recristalización a temperaturas relativamente bajas $[\underline{15}, \underline{16}]$. Este proceso interfiere con la precipitación, por lo que ocurre una competencia entre ambos. Estas nanopartículas pueden nuclear a partir de zonas de desorden de corto alcance y crecer mediante el mecanismo de Ostwald. Por tal motivo el pico de microdureza aparece cuando el tamaño promedio entre todas las partículas existentes es tal que el reforzamiento es máximo. Existe un tamaño para el cual se logra este máximo. Partículas muy pequeñas son cortadas, por lo que no favorecen de manera notable el endurecimiento del material, mientras que partículas mayores que cierto tamaño crítico provocan sobreenvejecido.
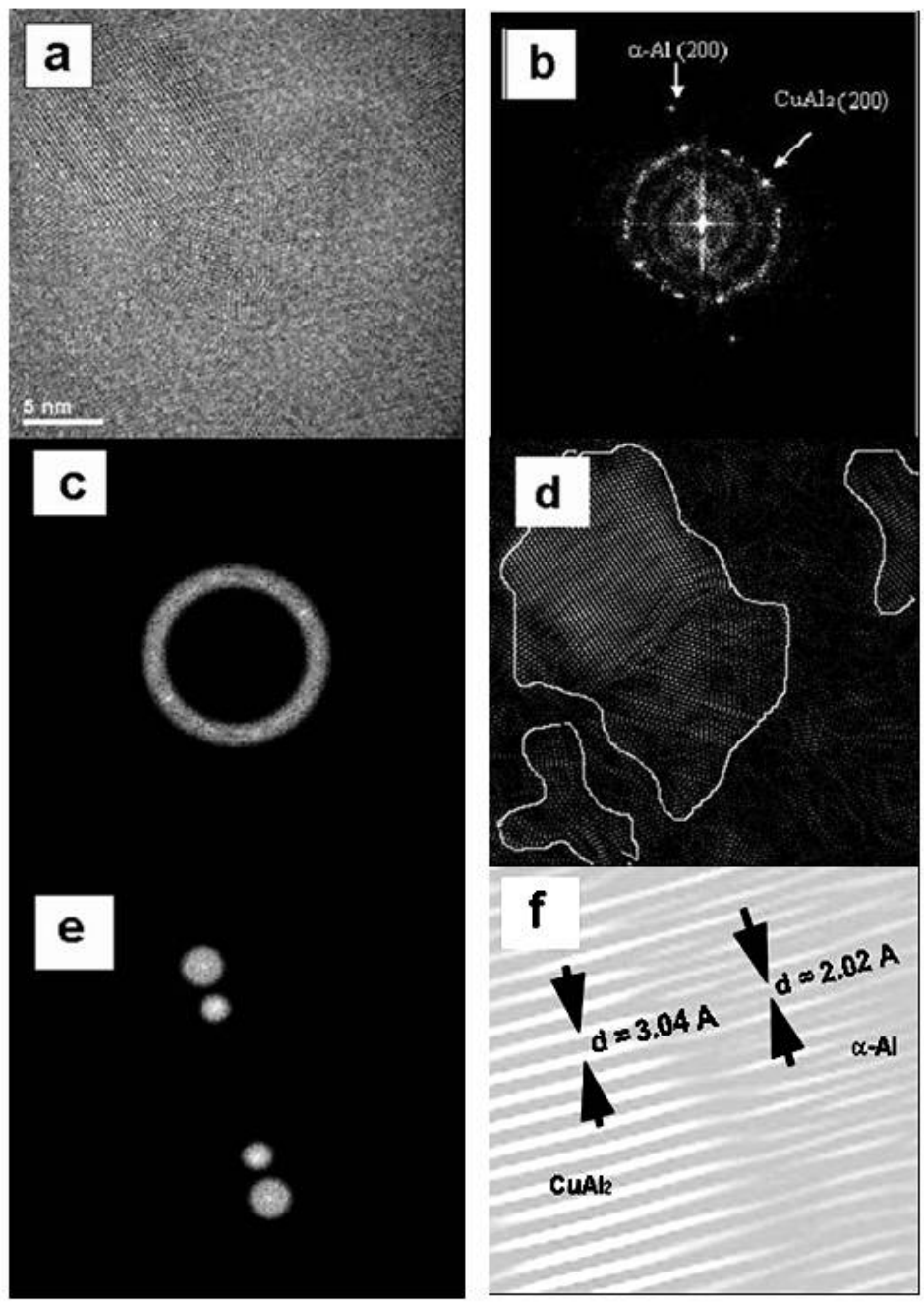

Figura 5: (a) Micrografía obtenida mediante HRTEM para las cintas envejecidas durante 40 h donde se pueden apreciar nanopartículas y diferentes dominios cristalinos (b)Transformada de Fourier donde se muestran las principales frecuencias de la matriz y la fase $\mathrm{CuAl}_{2}$, (c) Frecuencias correspondientes al $\mathrm{CuAl}_{2}$ utilizadas para el filtrado de imagen, (d)Imagen filtrada donde se muestran partículas de $\mathrm{CuAl}_{2}$,

(e) Frecuencias correspondientes al $\mathrm{CuAl}_{2}$ utilizadas para observar la interfase,

(f) Imagen filtrada ampliada donde se observa la coherencia matriz-precipitado. 


\section{CONCLUSIONES}

Después del análisis de los resultados se pudo llegar a las siguientes conclusiones:

1. El uso de solidificación rápida originó valores de microdurezas más de 2 veces superiores al presentado por las aleaciones obtenidas por fundición convencional.

2. La velocidad de solidificación no influyó sobre la composición química de los precipitados, pero si sobre su tamaño y forma.

3. El incremento en el contenido de Mg originó que se modificaran los precipitados formados. Para las aleaciones con $0.59 \%$ de $\mathrm{Mg}$ los precipitados formados presentan la composición química cercana al $\mathrm{CuAl}_{2}$, mientras que para las aleaciones con 3.80 y $6.78 \%$ de $\mathrm{Mg}$ se observó la presencia de precipitados correspondientes a la fase $\mathrm{Q}\left(\mathrm{Al}_{5} \mathrm{Cu}_{2} \mathrm{Mg}_{8} \mathrm{Si}_{6}\right)$.

4. Para las aleaciones obtenidas por solidificación rápida, el contenido de $\mathrm{Mg}$, la temperatura y el tiempo de envejecido influyeron de manera significativa sobre la microdureza. El aumento del contenido de $\mathrm{Mg}$ origina que las microdurezas sean mayores. Para estas aleaciones se observó el crecimiento de nanopartículas de $\mathrm{CuAl}_{2}$ y $\mathrm{Al}_{5} \mathrm{Cu}_{2} \mathrm{Mg}_{8} \mathrm{Si}_{6}$ existentes en el estado de colada y la recristalización originada por el alto contenido de elementos de aleación.

5. Para las aleaciones obtenidas por fundición convencional solo la temperatura influyó significativamente sobre la microdureza.

\section{AGRADECIMIENTOS}

Los autores quisieran expresar su agradecimiento a G. Lara y R.D. Cervantes por su asistencia técnica, así como a la CIC y la CGEP (UMSNH), México.

\section{BIBLIOGRAFÍA}

[1] HATCH, J.E., Aluminum, Properties and Physical Metallurgy, American Society for Metals, USA, 1993.

[2] Metals Handbook, $10^{\text {th }}$ Edition. Volume 2. Properties and Selection: Nonferrous Alloys and SpecialPurpose Materials, ASM International, 1990.

[3] WALTER, J.L., JACKSON, M.R., SIMS, C.T., Alloying, ASM International, 1988.

[4] HEUSLER, L., SCHNEIDER, W. "Influence of alloying elements on the thermal analysis results of Al-Si cast alloys", Journal of Light Metals, v. 2, n. 1, pp. 17-26, Feb. 2002.

[5] KOBAYASHI, T., ITO, T., YAO, Q., FATAHALIA, N., "Fatigue properties and microstructure of Al-Si$\mathrm{Cu}$ system casting alloys", Materials science and Technology, v. 15, n. 9, pp. 1037- 1043, Sept. 1999.

[6] MASAKAZU, H., SHINJI, Y., Patent US6059902. Aluminum alloy of excellent machinability and manufacturing method thereof. Kobe Steel Ltd (JP, 2000.

[7] OUELLET, P., SAMUEL, F.H., "Effect of Mg on the aging behaviour of Al-Si-Cu 319 type aluminium casting alloys", Journal of Materials Science, v. 34, n. 19, pp. 4671-4697, Oct. 1999.

[8] GAO, X., NIE, J.F., MUDDLE, B.C. "Effects of Si additions on the precipitation hardening response in the Al-Cu-Mg-(Ag) alloy", Materials Science Forum, v. 217-222, pp. 1251-1256, 1996.

[9] SAMUEL, F.H., OUELLET, P., SAMUEL, A.M., DOTY, H.W., "Effect of Mg and Sr additions on the formation of intermetallics in Al-6 wt pet Si-3.5 wt pct $\mathrm{Cu}-(0.45)$ to (0.8) wt pet Fe 319-type alloys", Metallurgical and Materials Transactions A, v. 29 A, n. 12, pp. 2871-2884, Dic. 1998.

[10] SAMUEL, F.H., PUCELLA, G., VILLENEUVE, C., SAMUEL, A.M., DOTY, H.W., VALTIERRA, S., "Microstructural observations on Fe-intermetallics in unmodified and $\mathrm{Sr}$-modified Al-Si-Cu (A380.1) die casting alloy", International Journal of Cast Metals Research, v. 12, n. 4 , pp. 197210, Jun. 1999. 
[11] LUTFI, M., UNLU, N., ERUSLU, N., GENC, A., "Characterization investigations of a melt-spun ternary Al-8Si-5.1Cu (in wt.\%) alloy", Materials Letters, v. 57, n. 21, pp. 3296-3301, Jul. 2003.

[12] BATRA, L.S., LAIK, A., KALE, G.B., DEY, G.K., KULKARNI, U.D., "Microstructure and properties of a Cu-Ti-Co alloy", Materials Science and Engineering A, v. 402, n. 1, pp. 118-125, Ago. 2005.

[13] LI, C., WANG, L., INOUE, A., "Precipitation of icosahedral quasicrystalline and crystalline approximant phases in $\mathrm{Zr}-\mathrm{Cu}-(\mathrm{Co}, \mathrm{Rh}$ or Ir) metallic glasses", Journal of Non-Crystalline Solids, v. 306, n. 2, pp. 175-181, Ago. 2002.

[14] JI, Q.G., GU, B.X., ZHANG, J.R., TIAN, Z.J., DU, Y.W., "Effects of aging at room temperature on asspun Nd2Fe14B/a-Fe nanocomposite magnets", Journal of Magnetism and Magnetic Materials, v. 288, pp. 84-91, Mar. 2005.

[15] Clavaguera-MORA, M.T., RODRIGUEZ-VIEJO, J., JACOVKIS, D., TOURON, J.L., CLAVAGUERA, N., HOWELLS, W.S., "Neutron diffraction and calorimetric study on Al-based metallic glasses", Journal of Non-Crystalline Solids, v. 287, n. 1, pp. 162-166, Jul. 2001.

[16] KUSY, M., RIELlO, P., BATTEZZATI, L., "A comparative study of primary Al precipitation in amorphous Al87Ni7La5Zr by means of WAXS, SAXS, TEM and DSC techniques", Acta Materialia, v. 52, n. 17, pp. 5031-5041, Oct. 2004. 